\title{
Angiosarcoma of the Thyroid Gland with Bilateral Malignant Pleural Effusion: A Case Report with Review of the Literature
}

\section{Vijaya Ganta, MD*; Gail Nunlee- Bland, MD; Robert L. Copeland Jr., Ph.D}

Division of Endocrinology, Diabetes and Metabolism; Howard University Hospital,

Washington $D C$, United States of America

*Corresponding Author: Vijaya Ganta MD, Division of Endocrinology, Diabetes and

Metabolism; Howard University Hospital, Washington DC, United States of America
Received: August 21, 2021

Published: September 06, 2021

(C) All rights are reserved by Vijaya Ganta

MD., et al.

\begin{abstract}
Objective: Our objective is to report a case of angiosarcoma of the thyroid gland with bilateral malignant pleural effusion.

Case Report: A 70-year-old African American woman with a medical history significant for Grave's disease status post radioactive iodine ablation, and postablative hypothyroidism for 39 years, presented with progressive hoarseness of voice and dysphagia for one year. Physical exam revealed diffuse enlargement of the thyroid, no thyroid bruit or cervical lymphadenopathy. Thyroid function tests TSH, FT3, and FT4 are normal and the thyroid peroxidase antibody was negative. The ultrasound of thyroid reported as a large thyroid gland with diffuse heterogeneity of the parenchyma with no clear-cut margins. The CT scan of the neck and chest with contrast reported as $7.5 \times 5.1 \mathrm{~cm}$ thyroid mass with ill-defined margins surrounding the trachea, moderate bilateral pleural effusion, pulmonary vascular congestion, bibasilar atelectasis, and/or infiltrates.

The fine needle aspiration of the thyroid under ultrasound guidance reported as angiosarcoma. Immunomarkers were positive for CD34 and CD31, and PAX-8, P53, and thyroglobulin reported as negative. Ultrasound guided pleural fluid aspiration analysis reported as angiosarcoma. Our Patient had total thyroidectomy with wide local excision. Pathology findings reported as high grade epithelioid angiosarcoma of the thyroid with tumor extension at surgical margins. The tumor cells showed strong CD31 immunopositivity, confirming the diagnosis of angiosarcoma. TTF-1 immunostain highlights an entrapped thyroid follicle within the tumor. Our patient was started on radiation therapy 4 weeks after surgery followed by chemotherapy-Taxol. Unfortunately, our patient passed away within 4 months of diagnosis.

Discussion: Angiosarcoma of the thyroid gland is mainly diagnosed by characteristic immunohistochemical features supported by immunopositivity for vascular endothelial markers such as CD31, CD34, factor VIII-related antigen, and immunonegativity for epithelial markers such as endothelial membrane antigen, pancytokeratin, and thyroglobulin. It has a relatively poor prognosis because it typically metastasizes to local lymph nodes and the lungs, and in later stages to the bone marrow. Treatment is wide local surgical excision. Radiation therapy may be effective in some patients and can be completed using chemotherapy.
\end{abstract}

Conclusion: Thyroid angiosarcoma can be highly aggressive with a poor prognosis.

\section{Abbreviations}

TSH: Thyroid Stimulating Hormone; FT3: Free Triiodothyronine; FT4: Free Thyroxine; CT: Computed Tomography; H\&E: Hematoxylin and Eosin.

\section{Introduction}

Angiosarcoma of the thyroid gland is a rare and highly aggressive malignant vascular endothelial tumor [1-3]. It is common in patients with a history of endemic goiter as a result of iodine de- 
ficiency, especially in the Swiss Alpine region [4,5]. In Northern Italy, Austria, and Switzerland, $2-10 \%$ of malignant thyroid tumors are angiosarcomas [6,7]. The most common age group affected are adults in their fifties to eighties (65 years is the median age) [6-8]. It is less common in men (women to men ratio 9:3) [7].

\section{Case Report}

A 70-year-old African American woman with a medical history significant for Grave's disease status post radioactive iodine ablation, and postablative hypothyroidism for 39 years, presented with progressive hoarseness of voice and dysphagia for one year. Physical exam revealed diffuse enlargement of the thyroid, no thyroid bruit or cervical lymphadenopathy. Thyroid function tests TSH, FT3, and FT4 were normal and the thyroid peroxidase antibody was negative. TSH was $3.95 \mathrm{mU} / \mathrm{L}(0.45-4.5)$, FT3 was $2.5 \mathrm{pg} / \mathrm{ml}$ (2-4.4), FT4 was $1.12 \mathrm{ng} / \mathrm{dl}$ (0.82-1.77), and the thyroid peroxidase antibody was $8 \mathrm{IU} / \mathrm{ml}(0-34)$. The ultrasound of thyroid reported as a large thyroid gland with diffuse heterogeneity of the parenchyma with no clear-cut margins.

The CT scan of the neck and chest with contrast reported as 7.5 x $5.1 \mathrm{~cm}$ thyroid mass with ill-defined margins surrounding the trachea, moderate bilateral pleural effusion, pulmonary vascular congestion, bibasilar atelectasis, and/or infiltrates.

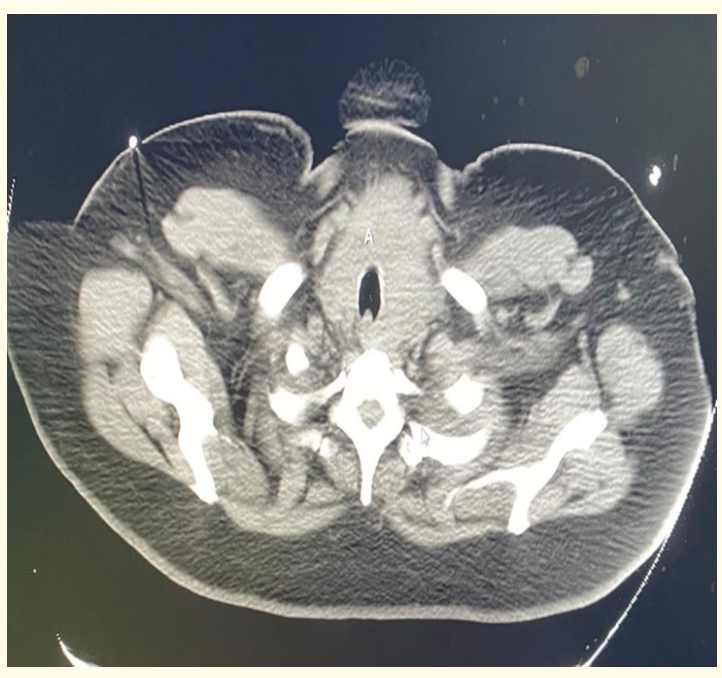

Figure 1: CT scan of neck with contrast transverse view revealed $7.5 \times 5.1 \mathrm{~cm}$ thyroid mass surrounding the trachea.

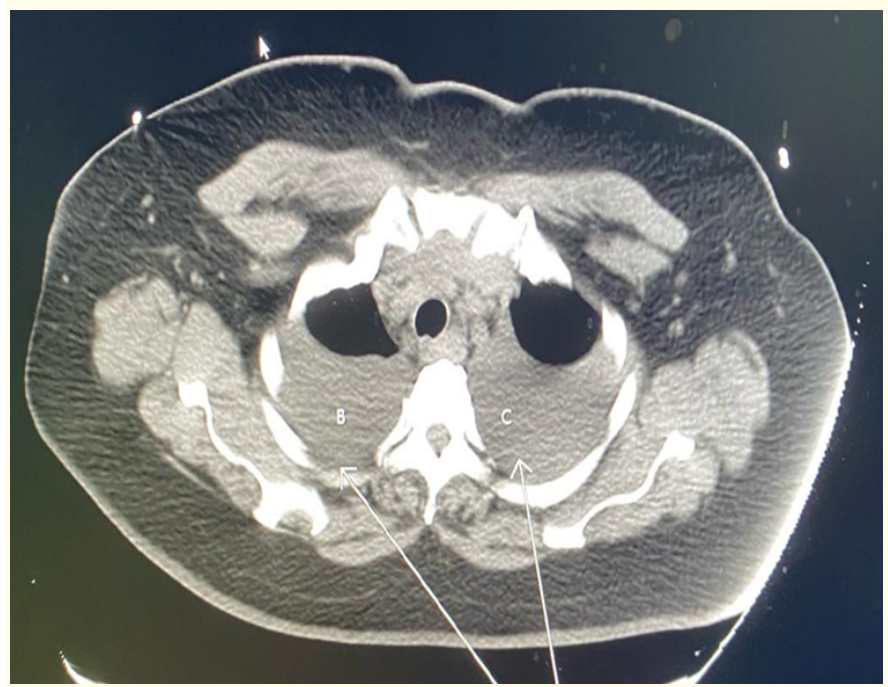

Figure 2: CT scan of chest with contrast transverse view revealed moderate bilateral pleural effusion.

The Bone scan reported as no metastasis. The Esophagogram reported mild esophageal dysmotility. The fine needle aspiration of the thyroid under ultrasound guidance reported as angiosarcoma. Immunomarkers were positive for CD31 and CD34, and PAX-8, P53, and thyroglobulin reported as negative. Ultrasound guided pleural fluid aspiration analysis reported as angiosarcoma. Our Patient had total thyroidectomy with wide local excision. Pathology findings reported as high grade epithelioid angiosarcoma of the thyroid gland with extensive tumor extension at surgical margins. Histologically H\&E staining showed sheets of malignant endothelial cells invading thyroid tissue with few entrapped thyroid follicles. The tumor showed anastomosing channels with occasional papillary fronds lined by epithelioid endothelial cells and highly pleomorphic vasoformative tumor cells with rudimentary vascular channels. The tumor cells showed strong CD31 immunopositivity, confirming the diagnosis of angiosarcoma. TTF-1 immunostain highlights an entrapped thyroid follicle within the tumor.

Our patient was started on radiation therapy 4 weeks after surgery followed by chemotherapy-Taxol. Unfortunately, our patient passed away within 4 months of diagnosis due to pulmonary complications. 


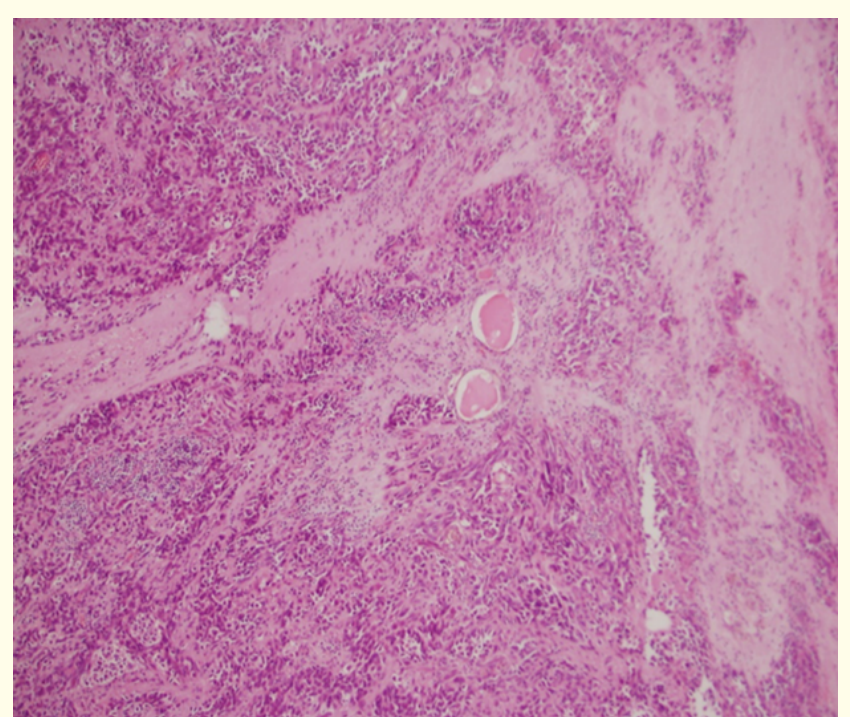

Figure 3: Sheets of malignant endothelial cells invading thyroid tissue with few entrapped thyroid follicles (arrow, H\&E, x40).

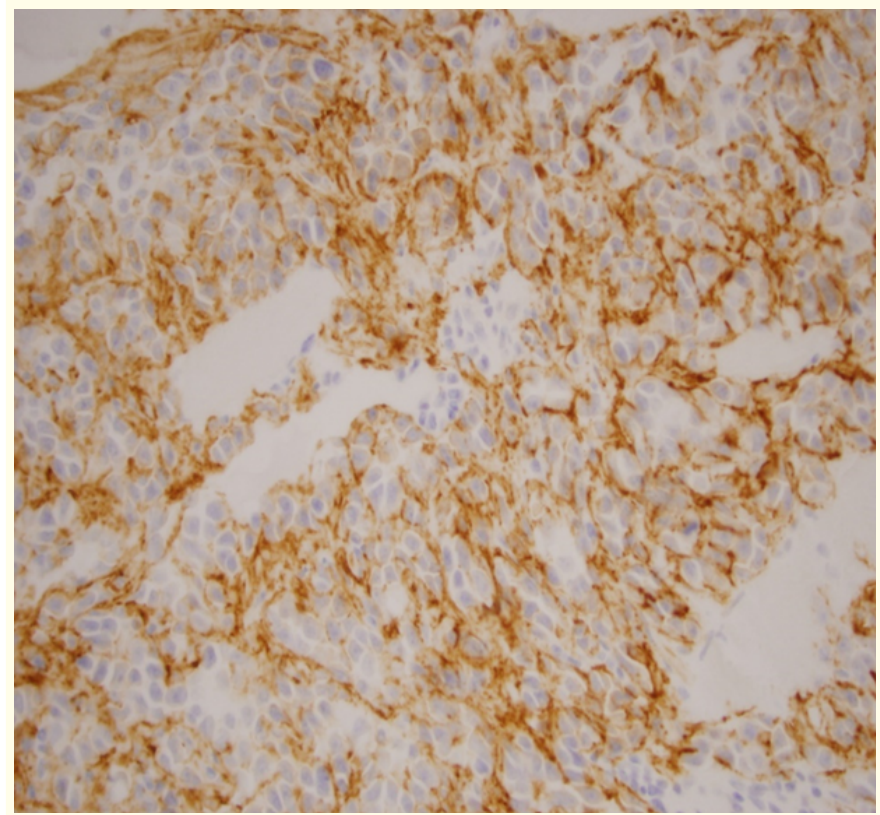

Figure 4: Tumor cells highlighted by CD 31 (x200).

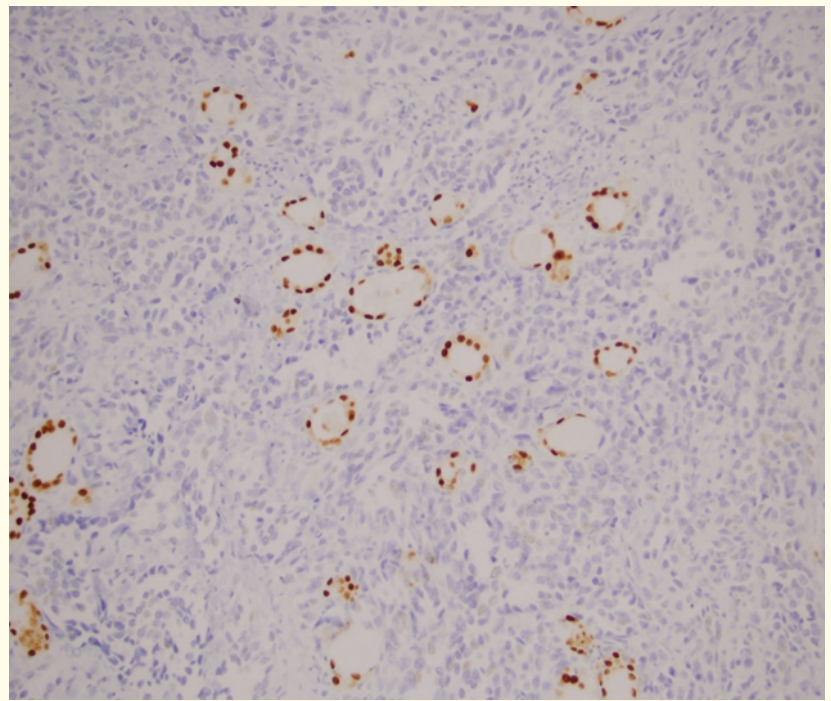

Figure 5: Entrapped thyroid follicles stained by TTF-1 (x200).

\section{Discussion}

The diagnosis of angiosarcoma of the thyroid can be difficult because the histological features may appear as other malignant vascular tumors. Our patient presented with 1-year progressive hoarseness of voice and dysphagia. Given morphological and immunohistochemical findings as well as the clinical history, a diagnosis of thyroid angiosarcoma with bilateral malignant pleural effusion was made. Our patient underwent thyroidectomy with wide local excision followed by radiation and chemotherapy. Unfortunately, the patient passed away with-in 4 months of the diagnosis due to pulmonary complications.

Angiosarcoma of the thyroid gland is mainly diagnosed by characteristic immunohistochemical features supported by immunopositivity for vascular endothelial markers CD31, CD34, factor VIII-related antigen, and negativity for epithelial markers such as endothelial membrane antigen, pancytokeratin, and thyroglobulin [9-12]. Most of these tumors can appear as poorly encapsulated and infiltrating masses. Local recurrence and metastasis can be common even after total excision of the tumor [13,14]. It has a relatively poor prognosis because it typically metastasizes to local lymph nodes and the lungs, and in later stages to the bone marrow 
[14]. Treatment is wide local surgical excision. Radiation therapy may be effective in some patients and can be completed using chemotherapy.

\section{Conclusion}

Angiosarcoma of the thyroid gland can be aggressive with distant metastasis and poor prognosis.

\section{Consent}

Written informed consent was obtained from the patient's family member for publication of the case report and any accompanying images.

\section{Conflicts of Interest}

The authors declare that there are no conflicts of interest.

\section{Sources of Support}

There are no sources of support.

\section{Acknowledgements}

A special thanks to Dr. Bonnie Davis and Dr. Hassan Nabhani for radiology images and Dr. Babak Shokrani for pathology slides.

\section{Bibliography}

1. De Lellis., et al. "Tumours of endocrine organs". In WHO Classification of Tumours. Geneva, Switzerland: WHO (2004): 113114.

2. Astl J., et al. "Hemangiosarcoma of the thyroid gland. A case report”. Neuro Endocrinology Letter 21 (2000): 213-216.

3. Malorana A., et al. "Angiomatoid carcinoma of the thyroid: clinicopathological analysis of seven cases from non-Alpine areas". Virchows Arch 429 (1996): 131-137.

4. Kalitova P., et al. "Angiosarcoma of the thyroid". European Archives of Otorhinolaryngology 266 (2009): 903-905.

5. Kaur A., et al. "Angiosarcoma of the thyroid: a case report with review of the literature". Endocrine Pathology 24 (2013): 156161.
6. Goh SG., et al. "Two cases of epithelioid angiosarcoma involving the thyroid and a brief review of non-Alpine epithelioid angiosarcoma of the thyroid". Archives of Pathology and Laboratory Medicine 127 (2003): 70-73.

7. Yilmazlar T., et al. "A case of hemangiosarcoma in thyroid with severe anemia due to bone marrow metastasis". Endocrine Journal 52 (2005): 57-59.

8. Petronella P., et al. "Primary thyroid angiosarcoma: an unusual localization”. World Journal of Surgical Oncology 10 (2012): 73.

9. Weiss SW and Goldblum JR. "Malignant vascular tumors". Enzinger and Weiss's Soft Tissue Tumors. Edited by: Weiss SW, Goldblum JR. 2008, Maryland Heights, MI, USA: Mosby (2008): 703-732.5.

10. Cutlan RT., et al. "Immunohistochemical characterization of thyroid gland tumors". Experimental and Molecular Pathology 69 (2000): 159-164.

11. Wang L., et al. "Clinicopathological features and prognostic factors in angiosarcoma: a retrospective analysis of 200 patients from a single Chinese Medical Institute". Oncology Letter 14.5 (2017): 5370-5378.

12. Sullivan HC., et al. "The utility of ERG, CD31 and CD34 in the cytological diagnosis of angiosarcoma: an analysis of 25 cases". Journal of Clinical Pathology 68.1 (2014): 44-50.

13. Rhomberg W., et al. "Malignant hemangioendothelioma of the thyroid gland: new results on pathogenesis, therapy and prognosis". Wiener klinische Wochenschrift 110 (1998): 479-484.

14. Rhomberg W., et al. "Prognosis and epidemiology of malignant hemangioendotheliomas of the thyroid gland". Schweizerische Medizinische Wochenschrift 123 (1993): 1640-1644.

Volume 5 Issue 10 October 2021 (c) All rights are reserved by Vijaya Ganta MD., et al. 